\title{
Artificial Immune System: State of the Art Approach
}

\author{
Prashant Kamal Mishra \\ M.Tech Student \\ Dept. of Computer Science \& Engg, \\ Ajay Kumar Garg Engineering College, UPTU, \\ Ghaziabad, India
}

\author{
Mamta Bhusry \\ Professor \\ Dept. of Computer Science \&Engg, \\ Ajay Kumar Garg Engineering College, UPTU, \\ Ghaziabad, India
}

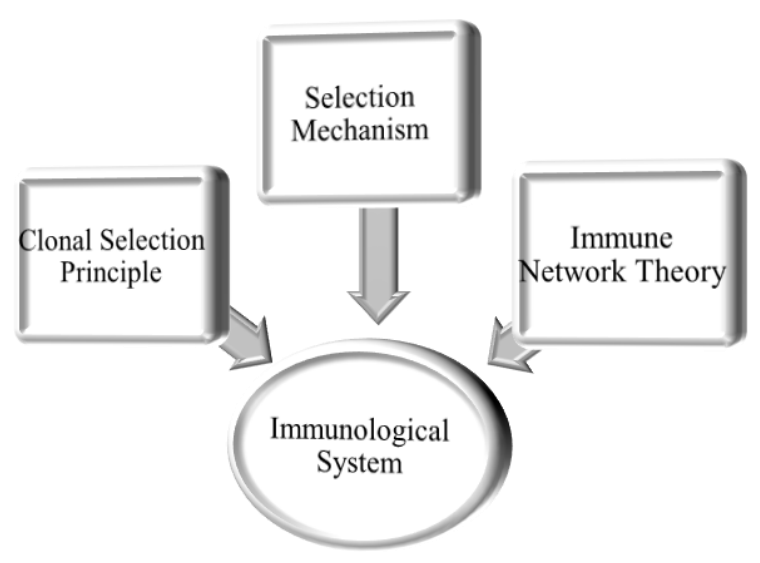

Fig.1 Principles of the Immune System

like building a bus which does not have a steering wheel models only an adaptive immune system - it will certainly go somewhere, but you have very little control.

\section{The Immune System: Metaphorically Speaking}

When problem solving capability comes under consideration, immune system should be the best process. It has the capability of learning, self-organization, adaptation, highly distributed and shows the memory. Some of the reasons are listed below by which computation from immune system is much popular:

(1) Recognition: If huge number of patterns is there then firstly immune system recognize them give response according to it. Moreover, the immune system has the capability to differentiate between and harmful nonself-cells and malfunctioning self-cells, thus immune system preserve some self-sense.

(2) Diversity: Diversity is another important characteristic of immune system. It involves two main process i.e. diversity generation and maintenance. In the generation phase receptor molecules are generated by recombining the gene segments. From any finite number of sets, many times the number of combinations can be generated so infinite number of receptors is generated from finite number of genes. In the maintenance phase immune cell reproduce themselves. For high diversity of immune receptor different pattern of cells required means high rate of somatic mutation.

(3) Distributed detection: Immune system has the natural distribution. There is not a single point by which full system is to be controlled. When any antigen is 
occurred in the system then specified cell is to be respond to that antigen.

(4) Metadynamics: The immune system is continuously generating new molecules and cells, and removing those cells that are not using from long time. The process of continuous creating and deleting the molecules and cells is known as Metadynamics.

(5) Self-regulation: in the immune system communication is done by neighborhood communication not by any single point. When any disease is identified then immediately immune system respond and encounter that disease and back to normal state, until new antigen is not to be identified. This Self-regulation mechanism is accounted explicitly in the immune network theory.

Moreover, this paper is formulated as follows: Section 2 gives the description of the "Biological systems"; Section 3 is describing existing AIS models and developed algorithms of immune system principles; Section 4 highlights the Hybrid intelligent system which provides the integration between AIS and other soft computing approaches; Section 5 presents applications of AIS and overall conclusion of this work.

\section{BIOLOGICAL KEY TERMS: DESCRIPTION}

\subsection{The Immune System}

A biological immune system comprises of molecules and cells that works cooperatively for establishing stable state of other system. The initial task of the biological immune system is to keep safe the body of human being from contaminated agents like viruses refer to as pathogens. A class of non-selfmolecules is known as antigen. Two mechanisms are there on which immune system work they are: Innate Immunity and Adaptive Immunity. When the pathogens are identified in the body the initial attack is done by the innate immune system and adaptive immunity is used for those pathogens that are not removed by innate immunity.

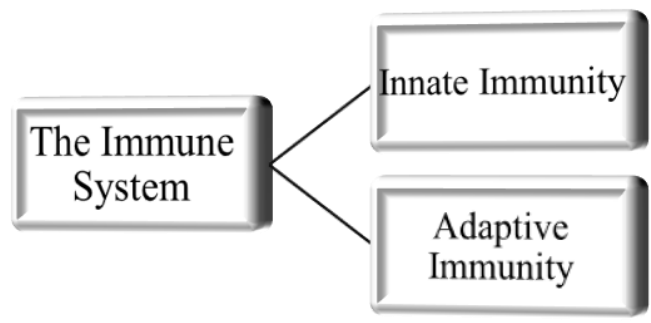

Fig.2Mechanism of the Immune System

Above fig. 2 explores the mechanism of the immune system which consists of innate and adaptive immunity which is explained further.

Innate immunity is a part of complete immune system that consist of cells and mechanism that prevent from infections by other organisms. Innate immune systems cell recognize and respond to pathogens in a comprehensive manner. It is an initial action performed by the innate cells so it does not guarantee to provide full protection.

Adaptive immunity empowers the body to identify and reply to any microbe by antigen receptor, even if it has never faced the invader before. It is more complex than innate immunity. It uses two types of lymphocytes (white blood cells) stated as
$\mathrm{T}$ cells and B cells. A random process takes place for generating antigen receptors. These cells are responsible for identifying and destroying antigens.

\subsection{Clonal Selection}

Burnet et al., [3] proposed the theory of clonal selection, and explained how immune system encounters the antigens and the way or process how the variants of lymphocytes are selected in order to eliminate antigens. He tells immunological memory as lymphocyte is cloned in to two types. One clone acts immediately to infection known as plasma cells and the other is long lasting which resides in the immune system for a longer period of time named as memory cells and it promotes a rapid secondary response.

\subsection{Negative Selection}

M. Ayara et al., [4] proposed the theory of Negative selection which shows that the body is protected from self-reactive lymphocytes. As there are no self cells responding still the immune system has the capability to find the unidentified antigens. When the generation of T-cells takes place, receptors undergoes a pseudo-random genetic rearrangement process. For negative selection process these receptors go through the censoring process in the thymus. The T-cells which are destroyed when responding to the self-proteins and the cells which do not respond to self-proteins are allowed to eliminate from the thymus. In order to protect the body from antigens, the $\mathrm{T}$ cells so generated are switching themselves in the system to accomplish immunological job.

\subsection{Immune Networks Theory}

Niles Jerne et al., [5] introduced the theory of immune network. Theory tells that the immune system is an interconnection network of lymphocytes and molecules. So, for antigen recognition, B cells make the interconnection network. B cells are connected in the way to make network stabilized. The strength of any two connected cells is exactly analogous to the confidence they dispense and these cells remain in connection until the confidence between those cells exceed a certain level of threshold value.

\section{ARTIFICIAL IMMUNE SYSTEM : LITERATURE REVIEW}

There are many other fields of AIS which are motivating the engineers and computer scientists to develop new algorithms. So, three main algorithms came into existence which have been steadily developed and achieve popularization:

(1) Clonal selection algorithms (CLONALG);

(2)Negative selection algorithms (NSA);

(3) Artificial immune networks (AINE).

Several applications of AISs have been successfully developed such as data mining, optimization, anomaly detection and computer security, etc. This section analyse the recent advancement done in the area of AIS. Many AIS applications and different types of models comes under it.

\subsection{Artificial Immune System Models}

\subsubsection{Clonal Selection Based Algorithms}

In 1959, Brunet proposed the clonal selection theory, in which when matching antigens are bonded together then lymphocytes are activated [3]. After lymphocytes are activated, its clones are generated in huge amount of numbers 
that will oppose the antigens. Self-bonded cells should be eliminated so no lymphocytes can bind to itself. When the process of the clonal selection of B-cells starts, the mean antibody affinity increases for the antigen and this process is known as affinity maturation. It is based on the reason that higher the affinity for the antigen, more fast the immune response will be. The selection of B-cells during the clonal expansion and affinity maturation is done by somatic hyper mutation. The outcome of antibodies is modified by somatic hyper mutation by doing some random changes to it.

Researchers have tried to draw some inspiration from the clonal selection process, in particular, the antigen affinity maturation process of B-cells along with the hyper mutation mechanism. Two motives of affinity maturation in B-cells have been drawn from a computational point [4]. Firstly, the generation of B-cells is compared to the affinity of the antigen that binds it, the higher the affinity, the more clones are produced. Secondly, the mutations suffered by B-cells are inversely proportional to the affinity of the antigen it binds. Utilizing these two features, the most popular clonal selection inspired algorithm, CLONALG came into existence [6].

There are various types of clonal selection algorithms are available in literature.

\begin{tabular}{|c|c|}
\hline Reference & Technique Explanation \\
\hline $\begin{array}{l}\text { L.N.de Castro et al [7] } \\
(2002)\end{array}$ & $\begin{array}{l}\text { Proposed this algorithm which is use for machine learning and pattern recognition and after doing some } \\
\text { changes in algorithm optimization task is also performed. CLONALG produces a number of N } \\
\text { antibodies, every antibody use for identify a solution for the learning and optimization. In each } \\
\text { computation process a number of good antibodies are select and perform the cloning and mutation } \\
\text { process for making the new antibody population. After performing the evaluation process best } \\
\text { antibodies are exchanged with the existing worst antibodies and new best antibody population is } \\
\text { created. }\end{array}$ \\
\hline Garett et al. [8] (2004) & $\begin{array}{l}\text { Analysed the CLONALG algorithm and tells the limitation of the accuracy of result. These limitations } \\
\text { are because of it requires several parameters and binary representation. It suggest some changes to the } \\
\text { algorithm like selection of mutation and the amount of clones. So the new algorithm is known as } \\
\text { Adaptive Clonal Selection (ACS) algorithm }\end{array}$ \\
\hline $\begin{array}{l}\text { Ruochen Liu et al.[9] } \\
(2004)\end{array}$ & $\begin{array}{l}\text { Proposed a new algorithm named as Adaptive Immune Clonal Strategy Algorithm (AICSA). Clonal } \\
\text { selection combines the global and local search so machine learning task can be easily done using } \\
\text { AICSA. It allocates the memory unit antibody population randomly }\end{array}$ \\
\hline Ying Yu et al. [10] (2004) & $\begin{array}{l}\text { Proposed the use of learning operator in the clonal selection algorithm. This learning operator is not } \\
\text { only increase the learning but also increase the detection efficiency }\end{array}$ \\
\hline $\begin{array}{l}\text { Felipe Campelo et al. [11] } \\
\text { (2005) }\end{array}$ & $\begin{array}{l}\text { Proposed A Real-Coded Clonal Selection Algorithm (RCSA) for numerical electromagnetic problems. } \\
\text { It is used for optimizing electromagnetic devices. It advices some changes in the clonal selection } \\
\text { algorithm for optimizing the real problem. RCSA has four parameters which has to be adjusted these } \\
\text { are: Population, no. of generation, rate of cloning, and cloning multiplying factor. }\end{array}$ \\
\hline $\begin{array}{l}\text { Vincenzo Cutello et al. } \\
\text { [12] (2005) }\end{array}$ & $\begin{array}{l}\text { Proposed an algorithm known as OPT-IA which solves global numerical optimization problem. Three } \\
\text { operators are used in this: cloning, hyper mutation and aging. Cloning is use to clone the B cells and } \\
\text { provide intermediate population. Receptor of B cell is done by hyper mutation and aging operator, this } \\
\text { operator removes the old population with new population previous B cells to avoid early convergence } \\
\text { and to improve diversity in current population. }\end{array}$ \\
\hline X.Bian et al. [13] (2005) & $\begin{array}{l}\text { An adaptive colonal algorithm for optimal phasor measurement unit (PMU) placement. It fixes the } \\
\text { probabilities of hyper mutation and recombination operators of the CLONALG algorithm and the } \\
\text { number of the cycle supplement population. These changes can help the optimization process. }\end{array}$ \\
\hline $\begin{array}{l}\text { Vincenzo Cutello et al. } \\
{[14](2006)}\end{array}$ & $\begin{array}{l}\text { Had described some changes in the OPT-IA algorithm which is known as opt-IMMALG (optimization } \\
\text { immune algorithm). Main changes in the algorithm are the use of real codes in place of the binary string } \\
\text { and inversely proportional hyper mutation operator is used in place of hyper mutation. }\end{array}$ \\
\hline M.Gong et al.[15] (2007) & $\begin{array}{l}\text { An improved clonal selection algorithm based on CLONALG with a novel mutation method, self- } \\
\text { adaptive chaotic mutation [18]. The min changes adopt the logistic chaotic sequence to generate the } \\
\text { initial antibody population, while the hyper mutation adopts self-adaptive chaotic mutation. }\end{array}$ \\
\hline M.Gong et al.[16] (2007) & $\begin{array}{l}\text { A Differential Immune Clonal Selection algorithm (DICSA) is proposed to solve the global } \\
\text { optimization problems [19]. It combines the clonal selection theory and differential evolution and } \\
\text { employs three operators: a clone operator, a differential mutation crossover mutation, and a standard } \\
\text { selection operator. }\end{array}$ \\
\hline $\begin{array}{l}\text { Dabrowski, J.et al. [17] } \\
\text { (2008) }\end{array}$ & $\begin{array}{l}\text { Gave an algorithm for solving the Graph Colouring Problem named as a parallel clonal selection } \\
\text { algorithm. In this the different processors are assigned to specific pool of antibody they parallel gives } \\
\text { the result so the performance is increased. }\end{array}$ \\
\hline H.Lu et al. [18] (2008) & $\begin{array}{l}\text { A Clonal Chaos Adjustment Algorithm (CCAA) is designed for Multi-modal Function Optimization. In } \\
\text { order to increase the overall convergence performance of CLONALG, it involves pros of the periodic } \\
\text { and dynamic principles of chaos system, and introduced the chaotic search technique into the } \\
\text { CLONALG to improve its search efficiency. }\end{array}$ \\
\hline
\end{tabular}




\section{A. Ciccazzoet al. [19] (2008)}

Purbasari, A. et al. [20] (2013)

Hongwei Dai et al. [21] (2014)

Yong Peng et al. [22] (2015)
Proposed the elitist Immune Programming (EIP), which gives the concept of elitism borrowed from other immune inspired algorithm; in which, at each step of generation $\mathrm{g}$, the best solution found cannot be eliminated from the population.

Designed an immune system using agent-based approach. The communication and performance of agents is define using UML diagrams. For checking or creating new AIS models this design is to be utilized.

Proposed Bi-direction quantum crossover-based clonal selection algorithm which is motivated by quantum theory in which energy is released when atom moves from higher to lower energy level and energy is absorbed when atom moves from lower to higher energy level. So, for increasing the performance it combines the Bi-direction quantum crossover and clonal selection.

Describe a new hybrid learning clonal selection algorithm (HLCSA), which is a combination of two learning approaches: Baldwinian learning and orthogonal learning. For global search Baldwinian learning is used and for local search orthogonal learning is used. This algorithm overcomes the problem of hypermutation operator which perturb antigen population which is not good for complex problems.

\subsubsection{Negative Selection Based Algorithms}

Natural immune systems have another type known as Negative Selection which is also very useful in developing the Artificial Immune systems. No self-cell is allowed so whenever T-cell identifies the self-cell, it is discarded before applying for immunological functions. Correspondingly, the detector works as T-cell, detector set are used to identify the self-samples in a group and delete them.

There is many application fields where Negative selection based algorithm is widely used, for example anomaly detection

\begin{tabular}{|l|l|}
\hline Reference & Technique Explanation \\
\hline S.Forrest et al. [23] (1994) & $\begin{array}{l}\text { Described a negative selection algorithm. In this firstly detector set are randomly generated by } \\
\text { creating candidates and then checks those elements which have self-data and eliminate them. } \\
\text { Finally these detectors can be used to find variance. } \\
\text { The NS Mutation algorithm defines somatic hyper mutation; which removes lay-offs and keeps } \\
\text { tunable constraints [4].It comprise of three steps: explain self-information, create cell detector and } \\
\text { Match the detector with self-information based on affinity threshold. }\end{array}$ \\
\hline $\begin{array}{l}\text { L.Gonzalez et al. [24] } \\
\text { (2004) }\end{array}$ & $\begin{array}{l}\text { A self-adaptive negative selection technique for anomaly detection uses self-adaptive techniques } \\
\text { for constraint tuning [24]. The main algorithm is divided in to two phases: the primary population } \\
\text { generation and the growth of the population. }\end{array}$ \\
\hline K.Lgawa et al. [25] (2009) & $\begin{array}{l}\text { Proposed an algorithm called as Artificial Negative Selection Classifier. Initially negative selection } \\
\text { algorithm is not able to solve the problem of over fitting and over searching. So, this algorithm } \\
\text { overcome these problems and increases the application area to multiclass classification. }\end{array}$ \\
\hline $\begin{array}{l}\text { Ilhan Aydin al. [26] (2010) } \\
\text { Maoguo gong et al. [27] } \\
\text { (2012) }\end{array}$ & $\begin{array}{l}\text { Proposed a new method named as Chaotic-based hybrid negative selection algorithm. It uses } \\
\text { better result for anomaly detection problem. }\end{array}$ \\
\hline $\begin{array}{l}\text { Presented an efficient negative selection for anomaly detection. In this self-detector are generated } \\
\text { using extra training. Main motive of extra training is to eliminate self-samples and reduce the cost } \\
\text { of computation at training stage. Self-region coverage is also improved by it. }\end{array}$ \\
\hline
\end{tabular}

\subsubsection{Artificial Immune Network Models}

\begin{tabular}{|l|l|}
\hline Reference & Technique Explanation \\
\hline Jerne (1974) & $\begin{array}{l}\text { Described the theory on immune network which is defines the idea and theory of immune network, } \\
\text { researchers develop the models for solving the problems in various field. }\end{array}$ \\
\hline Neal et al. [28] (2001) & $\begin{array}{l}\text { Proposed the system framed as Self-Stabilizing Artificial Immune System (SSAIS) for determining } \\
\text { time-varying data using Resource Limited Artificial Immune System (RLAIS). The difference } \\
\text { between previous and this algorithm is that there is unlimited number of resources are available. }\end{array}$ \\
\hline $\begin{array}{l}\text { Secker, A. et al. [29] } \\
\text { (2003) }\end{array}$ & $\begin{array}{l}\text { Presented a model for Email Classification named as Artificial Immune System for E-mail } \\
\text { Classification (AISEC). It is capable to differentiate between interesting and non-interesting emails } \\
\text { without the use of again and again training. }\end{array}$ \\
\hline Jia Lv et al. [30](2007) & $\begin{array}{l}\text { Proposed an algorithm for multimodal function optimization, previously solving the problem with } \\
\text { artificial immune system it may have some problems like premature convergence phenomena and } \\
\text { unsatisfying searching precision so to overcome this problem improved chaos immune network is } \\
\text { used. For premature convergence, stopping criterion is used and searching accuracy is improved by } \\
\text { using chaos variable. }\end{array}$ \\
\hline $\begin{array}{l}\text { Wenlong Huang et al. [31] } \\
\text { (2008) }\end{array}$ & $\begin{array}{l}\text { Proposed a network stated as Immune Kernel Clustering Network (IKCN). It works as a } \\
\text { is divided in subparts by the antibody and each subpart is mapped into high dimensional feature } \\
\text { space and each antibody neighborhood is regarded as a support vector hyper sphere. Results of } \\
\text { these support vectors are combined using the MST and global clustering solution is found. }\end{array}$ \\
\hline
\end{tabular}




\subsubsection{Other developed Algorithms}

\subsubsection{Conserved Self Pattern Recognition Algorithm (CSPRA).}

\begin{tabular}{|l|l|}
\hline Senhua Yu et al. [32] & $\begin{array}{l}\text { Proposed a new algorithm known as CSPRA which is simulated by the Pattern Recognition } \\
\text { Receptors (PRRs) Model. PRRs are a primal part of the immune system. They are expressed by } \\
\text { cells of the innate immune system to identify pathogen-associated molecular patterns (PAMPs). } \\
\text { PRRs model suspects that APCs are inactive until they are activated with the help of encoded PRR } \\
\text { that identifies conserved pathogenic patterns. So, it is clear that PRRs model adds an extra layer to } \\
\text { Self/Non-self-Model. Because PRRs model involves in CSPRA so it naturally involves negative } \\
\text { selection. However, the anomaly detection in CSPRA works when the result is combined from } \\
\text { negative selection of T-cell and self-pattern recognition of APC. When the dubious antigen is be } \\
\text { positively examined by T cell negative selection APCs the self-pattern recognition is to be } \\
\text { conducted. }\end{array}$ \\
\hline
\end{tabular}

\subsubsection{Toll-like Receptor (TLR) algorithm}

\begin{tabular}{|l|l|}
\hline & $\begin{array}{l}\text { Proposed a TLR algorithm that comprise of two categories of population of interacting cells named } \\
\text { as DC cells and T-cells. The DCs act as immature detectors for assembling antigens as input from } \\
\text { an antigen data store and process the sign for a particular amount of duration. The DC can be called } \\
\text { (2007) } \\
\text { as- mature if it receives sign during assembling of antigen, otherwise semi mature. Once the life } \\
\text { cycle of DC cell is finished, the DC is converted to a "lymph node" in which antigen produced by } \\
\text { DC is matched with T-cells. If the antigen produced by mature DC matches with the T-cell, the } \\
\text { state of T-cell is activated. And if the antigen produced by semi mature DCs matches with the T- } \\
\text { cell, the cells are removed from the population. }\end{array}$ \\
\hline $\begin{array}{l}\text { J.P. Twycross et al. [34] } \\
\text { (2007) }\end{array}$ & $\begin{array}{l}\text { Gave the term accession which is categorised as "peculiar" if the huge lot of activated T-cells is } \\
\text { generated. In TLR algorithm, the signs used are more homologous to PAMP signs which are } \\
\text { defined as binary signs: "sign ON" or "sign OFF". At an initial phase, an infectious sign catalogue } \\
\text { is generated to gather all possible sign values. To implement sign values, training and testing of } \\
\text { sign is performed with the help of antigen data. The TLR algorithm attains wrong positive rates of } \\
\text { 0.15 and right positive rates of 0.75 when it was calculated on a system for anomaly detection } \\
\text { problem. }\end{array}$ \\
\hline
\end{tabular}

\subsubsection{Complex artificial immune system.}

Proposed a model that comprise of five layers: Encountered Layer (Ag layer), Pre-Processing Layer (APC layer), MHC Layer, Competitive Layer (Th cell layer), Simulation-inhibition Layer (B cells layer).These layers work with some input and output which is named as antigen and anti-body respectively. If any antigen is presented in the body then it can be identified by two different waysFirstly, through direct recognition of B cell and secondly, through the APC layer processes. An antibody is stated as the contrast associated in the pattern of an input and the weights of B cells.

In comparison with the traditional binary immune systems, the CAIS has attributed a unique feature to identify transformation, such as translation or rotation of pattern. Pattern recognition problem was tested using CAIS for recognizing written digits and thus, showed good immune memory collection and noise tolerance capabilities.

\section{HYBRID SYSTEM}

The development of intelligent systems through Hybridization is one of the upcoming trends. These techniques are the combination of different soft computing procedures like fuzzy logics, evolutionary computation, artificial neural networks (ANN), genetic algorithms (GA) and artificial immune systems (AIS). The main motive is to overcome the limitations of individual algorithm by combining different learning and adaptation techniques by which we can achieve collaborative effects. In recent years intelligent systems are used for the development of large number of new intelligent system designs. Due to this, scientists have made hybrid system which is the combination of AIS and other soft computing methods.

Hajela et al. [36] combined the genetic algorithms with AIS. They show that how to improve the genetic search characteristics, specially, in the situation of dealing with design issues.

D.Dasgupta [37] had shown the comparison between AIS and artificial neural networks and also shows the comparative results of the artificial systems. 
Nasraoui et al.[38] showed the unreliability and fuzziness inbuilt in the process of matching between anti-bodies and antigens and to overcome this situation using Fuzzy AIS.

Vergas et al. [39] devised a combined system named as CLARINET. CLARINET is an immune network of classifiers which works on automated movement by amalgamating the advantages of classifier systems based on learning process, evolutionary algorithms, and a modular structure named as immune network model. It involves in computation and simulation of robotic navigation.

Ji-Qing Xian et al [40] introduced a clustering technique based on fuzzy logic for solving the problem that arises during fuzzy k-means clustering using the clonal selection algorithm.

V.K.Karakasiset al.[41] proposed a combined technique for data mining task that involves a combination of features of clonal selection and programming based on gene's expression and determining the outcomes in the form of prediction accuracy to be very good.

Jian $\mathrm{Fu}$ et al. [42] introduced a combined network of artificially developed immune system that is formed by the combination of swarm learning and elite-keeping. This simulation shows that this combined technique has small time complexity and immediate convergence, so it is an effective optimization tool.

Zhaohui Gan et al.[43] proposed a method which is a combination of clonal selection algorithm and gene expression programming known as clonal selection-gene expression programming (CS-GEP). It gives higher convergence speed and modelling precision.

Gu Danzhen et al.[44] presented a network based on fuzzy immune system named as (FaiNet) which has a characteristic that forms clusters of dynamic loads. It works on three algorithms: artificial immune network (AIN), minimal spanning tree (MST), and the classification approach.

\section{CONCLUSION}

Artificial immune system have many applications, a list of them are presented in this section. These applications have been summarized in particular manner i.e. based on types of application and the particular fields. There is a possibility that among different category some of them are overlapped. There is many fields where AISs have been widely used-Data mining, computer security and Networking, Automation and design, Optimization, Anomaly detection, Text processing, Bio-informatics, Pattern recognition, clustering and classification, etc.

In surveying recent work on the clonal selection algorithm and artificial immune networks, we recognized that majority of work have done on applications rather than expansion and advancement of the algorithms. This survey have been highlighted the recent applications of AIS. In the AIS field, negative selection algorithms are also increase their popularity due to computer security and fault detection problem solving capability. For improving the algorithms performance negative selection algorithms are typically focused on creating new detector generation scheme. Although, Artificial Immune System models have accomplished tremendous achievements in many application areas, still many theoretical problems are there which require the further analyse such as convergence and advancement of unified framework. The advancement of the AIS is not because of the encouragement of the principles and methods of biological immune; rather it is from the combination of different techniques of soft computing that includes neural network, genetic algorithm, and fuzzy logics. In future there is still more study requires for resolving complicated real world situations and applying them to more challenging application areas like classification and future prediction problems.

\section{REFRENCES}

[1] D. Dasgupta, J. Zhou and F. Gonzalez, "Artificial immune system (AIS) research in the last five years," proceedings of the IEEE Congress Evolutionary Computation (pp.123 - 130 vol.1, Year of Publication: 2003).

[2] Dipankar Das gupta, "An overview of artificial immune systems and their applications", In Dipankar Das gupta (Ed.), Artificial Immune Systems and Their Applications, (Berlin Heidelberg : Springer-Verlag, 1999, pp. 3-21).

[3] Burnet F.M., The Clonal Selection Theory of Acquired Immunity. Nashville, Vanderbilt University Press in 1959.

[4] Ayara M., Timmis J., Castro L. de, and Duncan R., Negative Selection: How to Generate Detectors. In 1st International Conference on Artificial Immune Systems, pp. 89-98, September, 2002.

[5] Jerne N. K., Towards a Network Theory of the Immune System, Annales Immunology, vol. 125C, pp. 373-389, 1974.

[6] L.N.d.Castroand J.Timmis,"Artificial Immune Systems as a Novel Soft Computing Paradigm". Soft Computing Journal, Vol.7, Issue 8, pp. 526-544, 2003.

[7] L.N.d.Castro and F.J.V.Zuben, Learning and optimization using the clonal selection principle, IEEE Transaction Evolutionary Computation, vol.6, no. 3,pp.239-251, Jun.2002

[8] S.Garrett, Parameter-Free Adaptive Clonal Selection, Congress on Evolutionary Computation, Vol: 1, pp.: 1052- 1058, 2004.

[9] L.Ruochen , D.Haifeng and J.Licheng, Immunity Clonal Strategies, Proceedings of Fifth International Conference on Computational Intelligence and Multimedia applications,( pages 290- 295 Year of Publication: 2003 ).

[10] Y.Yu and C.Hou, A Clonal Selection Algorithm By Using Learning Operator, Proceedings of the Third IEEE International Conference on Machine Learning and Cybernetics, Shanghai, (pages 2924 - 2929 vol.5 Year of Publication: 2004)

[11] F.Campelo, F.Guimaraes, H.Igarashi and J.Ramirez, A Clonal Selection Algorithm for Optimization in Electromagnetics, IEEE Transactions on Magnetics, VOL. 41, NO. 5, pp. 1736 - 1739, 2005.

[12] V.Cutello, G.Narzisi, G.Nicosia, and M.Pavone, An Immunological Algorithm for Global Numerical Optimization, Artificial Evolution: 7th International Conference, Evolution Artificielle, Springer, LNCS 3871:284-295, 2005 
[13] X.Bian and J.Qiu, Adaptive Clonal Algorithm and Its Application for Optimal PMU Placement, Proceedings of International Conference of IEEE on Communications, Circuits and Systems, ( pages: 2102-2106 Year of Publication: 2005)

[14] V.Cutello, G.Nicosia and M.Pavone, Real Coded Clonal Selection Algorithm for Unconstrained Global Optimization using a Hybrid Inversely Proportional Hyper mutation Operator, The 21st Annual ACM Symposium on Applied Computing, vol. 2, pp. 950-954, 2006.

[15] M.Gong, L.Jiao, L.Zhang, and W.Ma, Improved RealValued Clonal Selection Algorithm Based On A Novel Mutation Method, Proceedings of International Symposium on Intelligent Signal Processing and Communication Systems ( pages Year of Publication: 2007 ).

[16] M.Gong, L.Zhang, L.Jiao and W.Ma, Differential Immune Clonal Selection Algorithm, Proceedings of International Symposium on Intelligent Signal Processing and Communication Systems( pages Year of Publication: 2007 ).

[17] J.Dabrowski and M.Kubale, Computer Experiments with a Parallel Clonal Selection Algorithm for the Graph Coloring Problem, IEEE International Symposium on Parallel and Distributed Processing, pp.1-6, 2008.

[18] H.Lu and M.Zhichun, A Clonal Chaos Adjustment Algorithm for Multi-modal Function Optimization, Proceedings of the 27th Chinese Control Conference, pages , 2008 .

[19] A. Ciccazzo, P. Conca, G. Nicosia, G. Stracquadanio, An advanced clonal selection algorithm with ad hoc network-based hypermutation operators for synthesis of topology and sizing of analog electrical circuits, in 7th International Conference on Artificial Immune Systems, Phuket, Thailand, 2008.

[20] Purbasari, A., Iping, S.S. ; Santoso, O.S. ; Mandala, R. , Designing Artificial Immune System Based on Clonal Selection: Using Agent-Based Modeling Approach, in $7^{\text {th }}$ asia Modelling Symposium (AMS), pages 11-15, 2013.

[21] Hongwei Dai, Yu Yang, Hui Li, Cunhua Li, Bi-direction quantum crossover-based clonal selection algorithm and its applications, in Elsevier's Expert Systems with Applications, Volume 41, Issue 16, Pages 7248-7258, 2014.

[22] Yong Peng and Bao-Liang Lu , Hybrid learning clonal selection algorithm, Information Sciences, vol. 296(1) pages 128-146, 2015.

[23] S.Forrest, A. S.Perelson, L.Allen, and R.Cherukuri, SelfNonself Discrimination in a Computer. In Proceedings of IEEE Symposium on Research in Security and Privacy (pages 202-212 Year of Publication: 1994).

[24] L.Gonzalez and J.Cannady, A Self-Adaptive Negative Selection Approach for Anomaly Detection, Congress on Evolutionary Computation, Volume 2, Page(s): 1561 1568,2004
[25] K.Igawa and H.Ohashi, A Negative Selection Algorithm for Classification and Reduction of the Noise Effect, Application Soft Computation, 2008.

[26] Ilhan Aydin', Mehmet Karakose, Erhan Akin, Chaoticbased hybrid negative selection algorithm and its applications in fault and anomaly detection, in Expert Systems with Applications Volume 37, Issue 7, Pages 5285-5294, 2010.

[27] Maoguo Gong', Jian Zhang, Jingjing Ma, Licheng Jiao, An efficient negative selection algorithm with further training for anomaly detection, in Knowledge-Based Systems, Volume 30, June 2012, Pages 185-191, 2012.

[28] M.Neal, Met-Stable Memory in an Artificial Immune Network, Proceedings of the 2nd International Conference on Artificial Immune Systems, Springer (pages 168-180 Year of Publication: 2003).

[29] A.Secker, A.Freitas, and J.Timmis, AISEC: an Artificial Immune System for E-mail Classification, Proceedings of the Congress on Evolutionary Computation of IEEE, (pages 131-139 Year of Publication: 2003).

[30] J.LV, Study on Chaos Immune Network Algorithm for Multimodal Function Optimization, Fourth International Conference on Fuzzy Systems and Knowledge Discovery, Vol.3, On page(s): 684-689, 2007.

[31] Wenlong Huang', Licheng Jiao, “Artificial immune kernel clustering network for unsupervised image segmentation", in Progress in Natural Science, Volume 18, Issue 4, Pages 455-461,2008.

[32] S. Yu, D. Dasgupta, Conserved Self Pattern Recognition Algorithm, in: $7^{\text {th }}$ International Conference on Artificial Immune Systems, Phuket, Thailand, 2008.

[33] U. Aickelin, J. Green smith, Sensing Danger: Innate Immunology for Intrusion Detection, The University of Nottingham, Nottingham, UK, 2007.

[34]J.P. Twycross, Integrated innate and adaptive artificial immune systems applied to process anomaly detection, in: School of Computer Science, University of Nottingham, UK, Nottingham, 2007.

[35] W. Wang, S. Gao, Z. Tang, A complex artificial immune system, in: 2008 Fourth International Conference on Natural Computation, 2008.

[36] P.Hajela, J.Yoo, and J.Lee, GA based simulation of immune networks - applications in structural optimization. Journal of Engineering Optimization, no. 29, pp. 131-149, 1997

[37] D.Dasgupta, Artificial neural networks and artificial immune systems: Similarities and differences. In IEEE International Conference on Systems, Man and Cybernetics, Orlando, FL, pp. 873-878, 1997.

[38] Nasraoui O., Uribe C., Coronel C., and Gonzalez F., TECHNO-STREAMS: Tracking Evolving Clusters in Noisy Data Streams with a Scalable Immune System Learning Model. Proceedings of the Third IEEE International Conference on Data Mining,(page(s) 235242 Year of Publication: 2003). 
[39] Vergas P., Castro L. de, Michelan R., and F.Zuben, An Immune Learning Classifier Network for Autonomous Navigation, International Conference on Artificial Immune Systems, Edimburgo. Lecture Notes in Computer Science. Berlin Heidelberg: Springer-Verlag, v.2787. pp. $69-80,2003$.

[40] Xian J., Lang F., and Tang X., A Novel Intrusion Detection Method Based on Clonal Selection Clustering Algorithm, Proceedings of the $4^{\text {th }}$ International Conference on Machine Learning and Cybernetics, Vol. 6, On page(s): 3905- 3910 Vol. 6, 2005.

[41] Karakasis V. and Stafylopatis A., Data Mining based on Gene Expression Programming and Clonal Selection", 2006 IEEE Congress on Evolutionary Computation, Canada, July 16-21, on page(s): 514-521. , 2006.
[42] Fu J., Li Z., and Tan H., 2007. A Hybrid Artificial Immune Network with Swarm Learning. 2007 International Conference on Communications, Circuits and Systems (ICCCAS'07), 11-13 July, Kokura, on page(s): 910-914.

[43] Gan Z., Li G., Yang Z., and Jiang M., 2007. Automatic Modeling of Complex Functions with Clonal Selectionbased Gene Expression Programming. Third International Conference on Natural Computation (ICNC 2007), Haikou, 24-27 Aug., Volume: 4, On page(s): $228-$ 232 .

[44] Gu Danzhen, Ai Qian ; Chen Chen, "The application of artificial immune network in load classification", in the $3^{\text {rd }}$ Electric Utility Deregulation and Restructuring and Power Technologies of IEEE, Page(s): 1394 - 1398, 2008. 